\title{
Tactile Feedback for Mobile Interactions
}

\author{
Stephen Brewster, Faraz Chohan and Lorna Brown \\ Glasgow Interactive Systems Group, Department of Computing Science \\ University of Glasgow, Glasgow, G12 8QQ, UK \\ stephen@dcs.gla.ac.uk www.dcs.gla.ac.uk/ stephen
}

\begin{abstract}
We present a study investigating the use of vibrotactile feedback for touch-screen keyboards on PDAs. Such keyboards are hard to use when mobile as keys are very small. We conducted a laboratory study comparing standard buttons to ones with tactile feedback added. Results showed that with tactile feedback users entered significantly more text, made fewer errors and corrected more of the errors they did make. We ran the study again with users seated on an underground train to see if the positive effects transferred to realistic use. There were fewer beneficial effects, with only the number of errors corrected significantly improved by the tactile feedback. However, we found strong subjective feedback in favour of the tactile display. The results suggest that tactile feedback has a key role to play in improving interactions with touch screens.
\end{abstract}

\section{Author Keywords}

Tactile icons, Tactons, touch-screen buttons, mobility.

\section{ACM Classification Keywords \\ H5.2. [User Interfaces]: Haptic I/O.}

\section{INTRODUCTION}

This paper presents a study into the use of tactile feedback for an on-screen PDA keyboard where a stylus (or finger) is used to press the keys. Entering text on such keyboards is problematic as the keys are small (less than $1 \mathrm{~cm}^{2}$ on a PDA such as in Figure 1). Trying to do this whilst mobile makes interaction even harder as the PDA and stylus are both moving. Particularly difficult situations are on buses or trains, which can be very bumpy, yet these are situations where people often want to read/send email, browse Web sites, etc. on their way to work. The aim of our work was to look at the effects of tactile feedback from key presses with a stylus to see if performance could be improved.

\section{BACKGROUND}

There have been some good examples of the use of tactile displays to improve desktop interfaces. For example, Mackenzie and others have successfully shown that tactile

Permission to make digital or hard copies of all or part of this work for personal or classroom use is granted without fee provided that copies are not made or distributed for profit or commercial advantage and that copies bear this notice and the full citation on the first page. To copy otherwise, or republish, to post on servers or to redistribute to lists, requires prior specific permission and/or a fee.

CHI 2007, April 28-May 3, 2007, San Jose, California, USA.

Copyright 2007 ACM 978-1-59593-593-9/07/0004 ...\$5.00. feedback can improve pointing interactions [1] when using a mouse. Tactile cues can aid users in hitting targets such as buttons faster and more accurately.

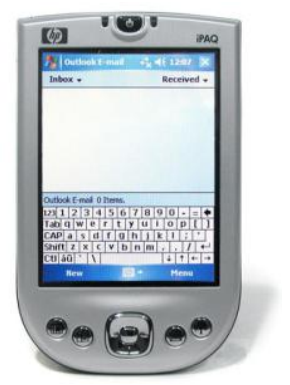

Figure 1: A typical on-screen keyboard on an HP iPAQ PDA.

However, most research in the area has focused on the design of tactile actuators; until recently there were few tactile actuators routinely available and they were often designed for use in different domains (e.g. sensory substitution systems). Lee et al. [7] developed a tactile stylus to use on touch screens and PDA's. Poupyrev et al. and Luk et al. [8, 9] have designed sophisticated tactile displays for handheld computers. Luk et al. have begun to look at interactions using their devices but none have been formally studied so there is little evidence that tactile displays are beneficial in practical situations.

Brewster and King [3] designed a tactile progress bar that indicated the progress of a download via the time difference between two tactile pulses; as the pulses got closer together the download got closer to completion. They found that users performed better with tactile progress bars than standard visual ones when involved in a visual typing task. Users were able to attend to the tactile feedback and type at the same time. In their experiment, the tactile actuator was on the user's wrist, but users were not mobile.

Brewster also looked at the benefits from adding sound to buttons for mobile interactions [2]. The aim was to overcome problems of contention for visual attention, where users must look where they are going when walking and cannot devote so much attention to the visual display. He found that sounds increased the amount of data people could enter on a PDA whilst walking and reduced subjective workload. We based the design of our vibrations on these sounds.

There has been very little work on the use of tactile displays in mobile settings. Many mobile devices already have vi- 
brotactile actuators built-in but they are little used in most interactions. The vibrations that accompany a mobile phone ringing are useful and alert the user to the call even if he/she cannot hear the audio. One problem can occur if users cannot feel the feedback (their phone maybe in a bag for example) but this is not the case with keyboard interactions as the user will be holding the device and so be in contact with the vibration.

The aim of our work is to see if tactile displays can offer other benefits for touch-screen devices. This paper presents two studies, one seated and one mobile, to investigate text entry on touch-screen displays. Text entry is a common activity and is based on button pressing, one of the most basic interaction techniques of all.

\section{REQUIREMENTS GATHERING}

The first stage of our work was to find out what kinds of errors people make when entering text on a PDA. We chose to investigate the interaction on an underground train as people use PDAs and phones on trains and buses every day whilst commuting. The underground is a good platform for testing as noise levels are very dynamic, being quiet when stopped at a station, but very noisy when the train is in motion. Light levels again vary dramatically. Vibration and movement are also very changeable. When the train is stopped there is little vibration. However, when it accelerates and decelerates people are subjected to lots of forces and vibration from the engine and general movement. Another important factor is that it is a safe environment as participants can be seated. Others have looked at testing whilst walking [2]. There are ethical issues as participants can trip or fall whilst taking part. All of these characteristics make it an interesting and realistic place to run studies.

We designed a simple interface to allow users to enter text using an on-screen keyboard on an iPAQ (copying the display seen in Figure 1). We gave participants short messages to type in and logged the kinds of errors and slips made. We encouraged them to be as fast and accurate as possible. There were six participants, all male students from the University (aged 19-26) and familiar with mobile devices. The study took around 10 minutes per person.

\section{Results and Discussion}

We saw a range of different errors occurring. One of the reasons was the competing attention demands of looking at the keys, the text entry window and the text to be entered. Analysis of keystroke logs showed a variety of problems:

Wrong letters: There were many examples of the wrong letters entered. Not all of these were caused by train movements, some were just mistakes. These are hard to detect and support with extra feedback as users may just tap the wrong key by mistake.

Slips: We noticed a number of slips, where the user put the stylus down on one letter and lifted it on another (the effect being no character is entered). Most slips occurred off the bottom or to the left of a key.

Double taps: We found that accidental double taps were common. This is due to the movements of the train, PDA and stylus, with the stylus bumping into the screen.

Many of the errors made were not corrected, again perhaps due to the attentional demands of the different parts of the display and the interference from the environment.

Our aim was to see if tactile feedback could overcome these problems. Audio feedback would be difficult in such an environment as it is very noisy; an earpiece would have to be worn. Visual feedback would be difficult as there is already much to look at and the screen is small. The tactile actuators in many PDAs and phones are not used unless a call is being received, so could provide extra feedback.

\section{EXPERIMENT 1: TACTILE DISPLAY IN THE LAB}

A first study investigated the part that tactile feedback might play in stationary interactions, which we tested in a laboratory. This would allow us to set a baseline of performance that we could then compare to a mobile setting.

The iPAQ we used for the study did not include a vibrotactile actuator so we added an external EAI C2 tactor (www.eaiinfo.com). This was connected via the headphone jack (see Figure 2). We placed it at the top right corner of the iPAQ so that the index finger of a right-handed user would rest on it. Other locations are possible, but for this study we were most interested in whether tactile feedback aided interaction, rather than studying actuator placement.
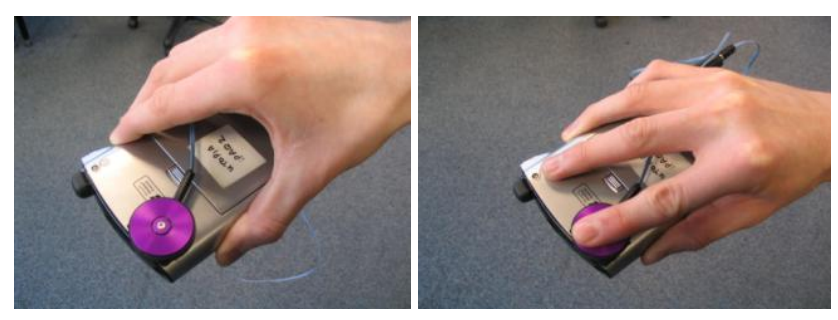

Figure 2: The vibrotactile actuator on the back of an iPAQ.

We used simple Tactons (tactile icons) [4] to represent different aspects of the button interaction. We kept the tactile design as simple as possible as keyboard interactions are fast and we needed our feedback to keep pace. We used two stimuli: one to indicate a successful button press and one to indicate an error. The success Tacton was played when a button was correctly pressed and then released. The error Tacton was played when a slip or double tap error occurred.

The design of the feedback was based on audio feedback added to buttons by Brewster [2]. We used an $800 \mathrm{~ms}$. $250 \mathrm{~Hz}$ sine wave success cue, and a rough (amplitude modulated) sine wave for the error cue. $250 \mathrm{~Hz}$ is in the range of greatest sensitivity of the skin [6] and the frequency at which the device resonates, giving the greatest power output. Brown et al. [4] showed that amplitude modulation is felt as 'roughness' and can provide a cue that 
is recognizably different to a 'smooth' sine wave, without taking any longer to play. These cues played as sound files through the tactile actuator.

We used 12 right-handed participants, all students from the University with no experience of touch screens. The study took place in a usability lab with participants seated, holding the iPAQ in their left hand. We used a within subjects design, with participants using both standard, visual buttons (Standard condition) and buttons with tactile feedback added (Tactile condition) in a counterbalanced order for 10 minutes each. A brief training phase preceded each condition to familiarise participants with the interface. Participants were given poems to type in and were asked to enter the text as fast and as accurately as possible. The software was similar to that used in the requirements capture, with a soft keyboard at the bottom of the screen and a text area at the top. Dependent variables were the amount of text entered, the total number of errors made (characters that were not in the poem) and the number of errors that were uncorrected by users.

\section{Results and Discussion for the Laboratory Study}

The results are shown in Figure 3. A T-test showed that there was a significant difference in the number of lines of text entered $\left(\mathrm{T}_{11}=6.28, \mathrm{p}<0.001\right)$ with more entered in the Tactile condition. Significantly more errors were made in the Standard condition $\left(\mathrm{T}_{11}=2.66, \mathrm{p}=0.02\right)$ and significantly more were corrected $\left(\mathrm{T}_{11}=4.10, \mathrm{p}=0.001\right)$ in the Tactile.

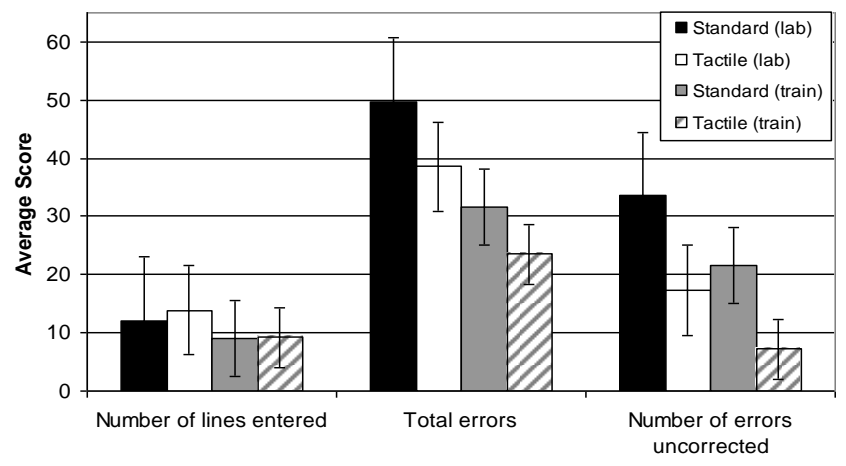

Figure 3: Results from the laboratory and mobile studies (with standard error bars shown).

The results show that with tactile feedback participants were generally performing much better: entering more text, making fewer errors and noticing more of the ones they did make. We suggest that the tactile feedback generally increased their awareness of mis-hit keys so that they could go back and correct them. There is room for improvement as they still missed some errors and better tactile feedback might bring this number down (although some of these are likely to be 'wrong letter' errors that we could not give extra feedback to support). The vibrations from the tactile feedback did not affect typing in a negative way as participants entered more text in the Tactile condition.

\section{EXPERIMENT 2: TACTILE DISPLAYS ON THE MOVE}

We ran the same experiment again but this time users were seated on a train on the Glasgow city underground. This allowed us to assess the effects of tactile feedback in a more realistic scenario, and if the benefits observed in the laboratory would carry over to the real world. We again used a within-subjects design to compare standard keyboard buttons to ones which we added tactile cues. The procedure and stimuli used in the experiment were as before to allow a comparison of the results. We used six new participants, again students from the University.

Participants sat in a seat on the underground train next to the experimenter who held the poem sheets (Figure 4). This time we also administered NASA TLX workload sheets after each condition [5]. We added an extra category of Annoyance to see how people felt about the extra feedback they received and whether the vibrations bothered them.

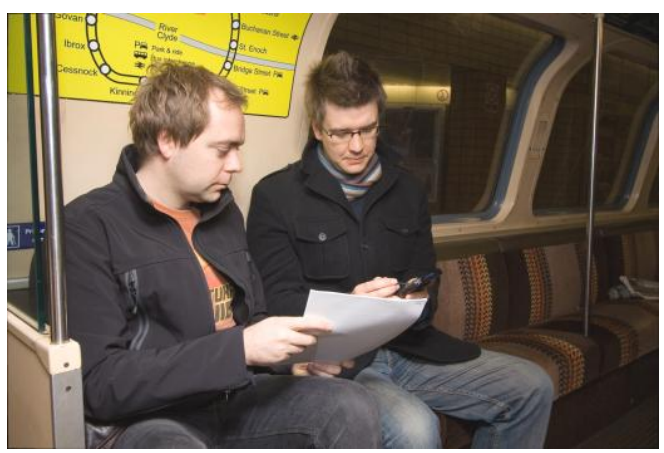

Figure 4: The experimental setup on the underground train.

\section{Results and Discussion for the Mobile Study}

Formal statistical analysis is limited due to the small number of participants, but gives some indication of where effects lie. The number of lines of text entered was not significantly different between the two conditions $\left(\mathrm{T}_{5}=0.34\right.$, $\mathrm{p}=0.74)$, neither was the total number of errors made $\left(\mathrm{T}_{5}=1.54, \mathrm{p}=0.18\right)$. There was, however, a significant difference in the number of uncorrected errors $\left(\mathrm{T}_{5}=3.06\right.$, $\mathrm{p}=0.02$ ), with more being corrected in the Tactile condition (as in the lab study). Figure 3 shows the results.

Results show that tactile feedback was less beneficial when users were mobile. The variations introduced by the environment (the underground generates much vibration) masked small benefits found in the lab (and the small number of participants will have caused more variance in the data). We did still see an effect for the number of uncorrected errors; more mistakes were still missed in the visual condition. This suggests that the extra feedback was still useful as correcting errors made is critical.

Figure 5 shows the results of the TLX questionnaires. Overall workload was significantly reduced $\left(\mathrm{T}_{5}=5.14\right.$, $\mathrm{p}=0.003$ ). A more detailed analysis showed significant reductions in workload in the Tactile condition for Mental Demand, Physical Demand, Effort Expended and Frustration (all with $\mathrm{p}<0.01$ ). There was a significant increase in 
perceived Performance Level $(\mathrm{p}<0.001)$ for the Tactile condition. There was no difference in terms of Time Pressure $(p=0.2)$. This is unsurprising as there were no differences between the two conditions in this respect. Annoyance was also found to be significantly reduced in the Tactile condition $(\mathrm{p}=0.006)$.

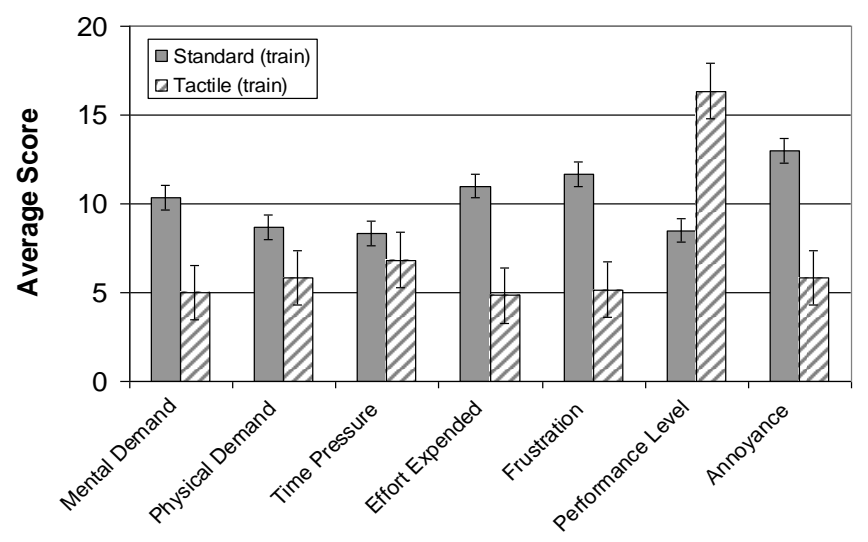

Figure 5: NASA TLX results for the mobile study.

The qualitative workload results show participants were strongly in favour of the Tactile condition, reducing almost all of the workload factors. This combined with the quantitative results shows that tactile feedback for touch-screen displays is beneficial in real mobile settings.

Comparing the results here to the laboratory study we can that the shapes of the graphs are broadly similar. There were $22.4 \%$ more errors made in the standard condition than the tactile when in the lab and $25.7 \%$ more when mobile. However, $48.3 \%$ more errors corrected in the tactile condition than the standard when in the lab but $66.9 \%$ more were corrected when mobile (the mean number of uncorrected errors in the tactile condition in the lab was 17.3 but only 7.1 when mobile). This suggests that the tactile feedback was even more beneficial for error correction in the mobile situation, giving participants useful information amongst all of the noise and vibration of the train. It is important that errors that are made are corrected; ideally fewer errors would occur, but if they do occur then it is crucial that the user notices and corrects them otherwise incorrect messages could be sent.

The results for the mobile study match some of those Brewster found with sonically-enhanced buttons when tested on the move (in that case with users walking) [2]. For example, he also found more data was entered when extra feedback was given. Another similarity was a large reduction in workload with the extra feedback when users were mobile. This suggests that touch-screen buttons are hard to use in mobile settings and users benefit when they are given extra assistance. The advantage of tactile over sound is that it can be given even in noisy environments. A future study will directly compare audio, tactile and a combination of the two feedback types to see which is most beneficial.

\section{CONCLUSIONS}

The studies presented here have shown that tactile feedback provides significant benefits for keyboard interactions on touch-screen devices, both in static situations and more dynamic, mobile ones. Such feedback is likely to help all button interactions on touch-screens, not just text entry, which would be a considerable benefit as buttons are very common. Giving tactile feedback via the device rather than the stylus also means that users would get the benefits even if using a finger to press the buttons.

Brewster [2] showed that sonic enhancement of buttons could improve performance. The downside of his solution was that sounds could be intrusive or not heard in noisy environments. Tactile feedback is an effective alternative and does not suffer the same drawbacks. A key recommendation from this work is for PDA and smart phone designers to use tactile feedback in more of the interactions with their devices as an easy way to improve usability.

\section{ACKNOWLEDGMENTS}

This work was funded EPSRC Advanced Research Fellowship GR/S53244.

\section{REFERENCES}

1. Akamatsu, M., MacKenzie, I.S. and Hasbrouq, T. A comparison of tactile, auditory, and visual feedback in a pointing task using a mouse-type device. Ergonomics, 38. 816-827.

2. Brewster, S.A. Overcoming the Lack of Screen Space on Mobile Computers. Personal and Ubiquitous Computing, 6 (3). 188-205.

3. Brewster, S.A. and King, A.J., An Investigation into the Use of Tactons to Present Progress Information. In Proc. of Interact 2005, Springer, 6-17.

4. Brown, L.M., Brewster, S.A. and Purchase, H.C., A First Investigation into the Effectiveness of Tactons. In Proc. of Worldhaptics 2005, IEEE Press, 167-176.

5. Hart, S.G. and Wickens, C. Workload assessment and prediction. In Booher, H.R. ed. MANPRINT, an approach to systems integration, Van Nostrand Reinhold, New York, 1990, 257-296.

6. Klatzky, R. and Lederman, S. Chapter 6: Touch. In Healy, A. and Proctor, R. eds. Handbook of Psychology Volume 4: Experimental Psychology, John Wiley and Sons, 2003, 147-176.

7. Lee, J.C., Dietz, P., Leigh, D., Yerazunis, W. and Hudson, S.E., Haptic Pen: A Tactile Feedback Stylus for Touch Screens. In Proc. of ACM UIST 2004, ACM Press Addison-Wesley, 291-294.

8. Luk, J., Pasquero, J., Little, S., MacLean, K., Levesque, V. and Hayward, V. A role for haptics in mobile interaction. In Proc. ACM CHI 2006, ACM Press Addison Wesley, 171-180.

9. Poupyrev, I. and Maruyama, S., Tactile Interfaces for Small Touch Screens. In Proc. of UIST 2003, ACM Press, 217-220. 